\title{
Measurements of $\phi$ Mesons Reconstructed from Hadronic and Leptonic Decays by the PHENIX Experiment at RHIC
}

\author{
Shengli Huang (for the PHENIX Collaboration) \\ Vanderbilt University - Dept of Physics and Astronomy \\ Nashville, TN 37235
}

\begin{abstract}
In this paper, we present the PHENIX preliminary results on the $\phi$ mesons mass and width, reconstructed from the $K^{+} K^{-}$decay in $\mathrm{Au}+\mathrm{Au}$ collisions at $\sqrt{S_{N N}}=200 \mathrm{GeV}$. The production of $\phi$ mesons in $200 \mathrm{GeV} \mathrm{Au}+\mathrm{Au}$ collisions has also been studied in both the $K^{+} K^{-}$and the $e^{+} e^{-}$decay channels. The yield and effective temperature obtained from invariant transverse mass $\left(m_{T}\right)$ spectra are studied and compared as a function of centrality in both decay channels.
\end{abstract}

\section{Introduction}

An extremely dense and hot matter with hints of partonic properties has been generated at RHIC using ultra-heavy ion collisions [2]. The mass and width of $\phi$ mesons may change in this medium [3]. Since the $\phi$ meson is the lightest $s \bar{s}$ bound state and its mass is close to two times the kaon mass, even a small mass shift will cause a large change in the branching ratio of $\phi \rightarrow e^{+} e^{-}$and $\phi \rightarrow K^{+} K^{-}$. Thus, comparison of the yield in these two decay channels provides a sensitive probe to study the properties of the medium. It also could provide information about the partial chiral symmetry restoration and help us understand the origin of mass. In the paper, we present the $\phi$ meson mass, width and their centrality dependence in $200 \mathrm{GeV} \mathrm{Au}+\mathrm{Au}$ collisions. We also present the $\phi$ mesons yield obtained in the $e^{+} e^{-}$and the $K^{+} K^{-}$decay channels from the same energy and collision system.

\section{Analysis Method}

The PHENIX experiment [4] has two central spectrometers, which cover $90^{0}$ in azimuth and pseudorapidity of $|\eta|<0.35$. Each arm can measure the momentum of charged particles produced in RHIC collisions using the Drift Chamber (DC) and the first layer of the Pad Chamber (PC1). The Beam-Beam Counters (BBC) in combination with Zero Degree Calorimeters (ZDC) provide the trigger and are used to determine the z-coordinate of the collision vertex and the event centrality. PHENIX also has a 130 ps timing resolution TOF subsystem covering half of the East arm and a 500 ps timing resolution Electromagnetic Calorimeter (EMCal) covering both arms. The TOF and EMCal identify kaons within $0.3<p(\mathrm{GeV} / \mathrm{c})<2.0$ and $0.3<p(\mathrm{GeV} / \mathrm{c})<1.0$ respectively. Electrons are identified with the Ring Imaging Cherenkov Detector $(\mathrm{RICH})$ and EMCal. To reconstruct the $\phi$ meson using the $e^{+} e^{-}$and the $K^{+} K^{-}$decays, we combine oppositely charged identified particles to form invariant mass distributions containing both the signal and combinatorial background. The shape of the combinatorial background is estimated by the mixed event technique, where particles are taken from different events which have similar multiplicity and collision vertex. The mixed event invariant mass distribution is normalized to $2 \sqrt{N_{++} N_{--}}$, where $N_{++}$and $N_{--}$are the measured integrals of like sign yields [5]. 



Figure 1: The centrality dependence of the $\phi$ meson mass and width in $200 \mathrm{GeV} \mathrm{Au}+\mathrm{Au}$ collisions

Raw yields are counted around known particle masses after subtraction of invariant mass distributions from mixed events. To study the $\phi$ mesons mass and width, we use a Breit Wigner convolved with a Gaussian function to fit the $\phi$ meson invariant mass distribution. The Gaussian function is implemented to take into account the finite mass resolution of the detector which has been determined to be about $1.4 \mathrm{MeV} / \mathrm{c}^{2}$ by the simulation.

\section{$3 \quad$ Results and Discussions}

Fig. 1 shows the mass and width of $\phi$ mesons obtained from $200 \mathrm{GeV} \mathrm{Au+Au} \mathrm{collisions}$ in the $K^{+} K^{-}$decay channel. The left plot shows the mass of the $\phi$ mesons as a function of the number of nucleons participating in the collisions. The systematic errors are represented by two lines. The systematic errors are mainly due to the uncertainty in the magnet field. The simulation study indicates that this field uncertainty will cause about $0.9 \mathrm{MeV}$ mass uncertainty. The right plot shows the mass width of the $\phi$ mesons as a function of the number of participants. The statistical errors are shown with blue lines and the systematic errors are shown as shadowed boxes. The systematic errors were evaluated by varying the normalization method and the fit region in the invariant mass distributions. The results shown in Fig.1 indicate that there is no centrality dependence of the $\phi$ mesons mass and width, and that the measurements are consistent with the PDG 
value.

Fig.2 shows the yield of $\phi$ mesons obtained at mid-rapidity from the $e^{+} e^{-}$and $K^{+} K^{-}$ decays in $200 \mathrm{GeV} \mathrm{Au}+\mathrm{Au}$ collisions. The two solid squares show the $\phi$ meson yield from the $e^{+} e^{-}$decay at centrality of $40-92 \%$ and $20-40 \%$. The yield is obtained by fitting the invariant transverse mass $\left(m_{T}\right)$ spectra with an exponential function. The yield from the $e^{+} e^{-}$decays in the most central collisions is shown as triangle and the minimum bias result is shown as open square. The solid circles show the $\phi$ meson yield from the $K^{+} K^{-}$decays at different centralities. The systematic errors come primarily from the choice of the normalization method and the mass window in which the raw yield is counted. As Fig.2 shows, the yield obtained from the $K^{+} K^{-}$and $e^{+} e^{-}$decays are similar and are consistent with each other within the quoted systematic errors. No significant difference is observed. However, we note that the life time of $\phi$ meson is around $40 \mathrm{fm} / \mathrm{c}$. Only low momentum $(<1 \mathrm{GeV} / \mathrm{c}) \phi$ mesons are expected to decay inside the medium. So, a low momentum study of the mass centroid and width as well as a direct yield comparison between the two decay channels is desirable. The background in the low momentum region in the $\phi \rightarrow e^{+} e^{-}$decay channel is prohibitively large. This measurement will become possible with the PHENIX hadron blind detector (HBD) upgrade, which will suppress the background by a factor of 30 in $e^{+} e^{-}$decays. After the upgrade, it will be possible to test whether the branching ratio changes in the presence of the medium. Fig.3 shows the inverse slope (effective temperature) obtained from an exponential fit to the invariant transverse mass $\left(m_{T}\right)$ spectra of $\phi$ mesons from $K^{+} K^{-}$and $e^{+} e^{-}$decays. The solid circles are the $K^{+} K^{-}$decay results and the square data points are the $e^{+} e^{-}$decay results. The effective temperature shows little or no centrality dependence in both decay channels. The two results are consistent within errors. One may expect that the inverse slopes will differ, if the decay kaons were affected strongly by hadronic re-scattering. However within the present errors, we do not see such an effect.

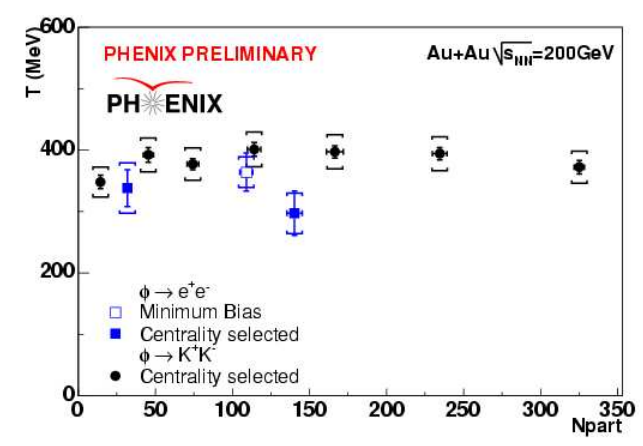

Figure 3: The centrality dependence of the effective temperature from the $\phi$ meson invariant $m_{T}$ spectra in $200 \mathrm{GeV} \mathrm{Au+Au}$ collisions

\section{Conclusions}

The PHENIX experiment has measured the production of $\phi$ mesons using their decays into $K^{+} K^{-}$and $e^{+} e^{-}$. The mass and width of the $\phi$ mesons using the $K^{+} K^{-}$decay channel were found to be independent of the centrality of the collisions and consistent with the PDG values. The integrated yield and effective temperatures obtained from the $K^{+} K^{-}$and the $e^{+} e^{-}$decay channels are consistent with each other at different centralities within the large systematic errors. In the future, the new HBD detector will help us to refine these measurements by suppressing the large background in the $e^{+} e^{-}$decay channel. 


\section{Acknowledgments}

This research is sponsored by the Division of Nuclear Physics, U.S. Department of Energy, under contract DE-FG05-92ER40712 and OJI grant DE-FG02-04ER41333. The complete list of PHENIX acknowledgments is provided in Ref [6].

\section{References}

[1] Slides:

http: //indico. cern. ch/contributionDisplay. py? contribId=94\&sessionId=7\&conf Id=9499

[2] Phenix Collab., K. Adcox et al., Nucl. Phys. A 757 (2005) 184 [arXiv:nucl-ex/0410003].

[3] G.E. Brown and M. Rho, Phys. Rev. Lett. 66 (1991) 2720; T. Hatsuda and S.H. Lee, Phys. Rev. C 46 (1992) 34; E. Oset and A. Ramos, Nucl. Phys. A 679 (2001) 616 [arXiv:nucl-th/0005046]; D. Cabrera and M.J. Vicente Vacas, Phys. Rev. C 67 (2003) 045203 [arXiv:nucl-th/0205075]; F. Klingl, T. Waas and W. Weise, Phys. Lett. B 431 (1998) 254 [arXiv:hep-ph/9709210].

[4] Phenix Collab., K. Adcox et al., Nucl. Instrum. Meth. A 499 (2003) 469.

[5] Phenix Collab., A. Toia et al., Nucl. Phys. A 774 (2006) 743 [arXiv:nucl-ex/0510006].

[6] Phenix Collab., A. Adare et al., Phys. Rev. Lett. 97 (2006) 252002 [arXiv:hep-ex/0609010]. 